Japanese Psychological Research

1979 , Vol. 21, No. 4, 18!-188

\title{
EFFECTS OF ONE EYE REMOVAL AT BIRTH ON RETENTION OF A VISUAL HABIT IN THE ALBINO RAT
}

\author{
FUMIO YAGI ${ }^{1}$ AND MAKOTO SAKAI \\ Netropsychology Laboralory, Saga Medical School
}

\begin{abstract}
Rats with one cye cnuclcated at birth (OEB group) were trained on a blackwhite discrimination task at three months of age. As a control, rats enucleated at three months of age were trained on the same task either immediately (Control group 1) or thee months (Control group 2) after enucleation. After reaching the criterion of learning, the rats of all three groups received the visual cortex lesion ipsilateral (IP lesion) or contralateral (CT lesion) to the remaining eye and were retrained. The results indicate that (a) with the IP lesion all three groups showed a substantial amount of retention, and (b) with the CT lesion the two control groups showed a complete loss of the habit, whereas the OEB group showed a certain amount of retention. These findings are discussed in relation to histological and electrophysiological observations made on the visual system when one eye is enucleated shortly after birth and after full devclopment.
\end{abstract}

Lund, Cunningham and Lund (1973) have reported that following removal of one eye within about ten days after birth, an increased number of optic nerve terminals became noticeable in the lateral geniculate body (LGB) and in the superior colliculus (SC) ipsilateral to the remaining eye in the albino rat. A similar type of observation was made by Hickey (1975) in that when one eye was enucleated within seven days after birth, the uncrossed optic fibers were obscrved to invade layer $A$ of the LGB. While these two studies suggest different mechanisms governing the phenomenon, that is, the former attributing it to aberrant uncrossed optic fibers and the latter to sprouting of normally existing uncrossed axons, they both agree on the point that such a phenomenon could not be observed when enucleation was performed at maturity. One naturally wonders whether or not these increased ter-

1 Requests for reprints should be sent to Fumio Yagi, Neuropsychology Laboratory, Saga Medical School, 421-6 Nabeshima, Nabeshima-machi, Saga, 840-01 Japan. minals are functional in the LGB and SC. Sakai (1978) suggests that they are functional in the LGB. Upon stimulating the optic nerve of the remaining eye, he was able to record a well shaped postsynaptic component in the ipsilateral LGB only in rats with one eye removed at birth but hardly ever in rats with one eye enucleated at three months of age. This finding indicates that by removing one cyc at birth the uncrossed retinal input coming from the remaining eye activates a greater number of neurons in the LGB. In other words, it can be said that the number of functionally effective optic terminals in the LGB increases as a result of removing one eye at birth.

It is well known that the visual system of the albino rat has one unique charactcristic: Only a few percent of the optic nerves do not cross at the chiasm but rather they project to the ipsilateral visual centers (Lund, 1965). This peculiar anatomical arrangement has led a number of investigators to study the functional significance of these uncrossed optic fibers in visually guided behavior (Creel \& 
Sheridan, 1966; Nadel \& Burcšová, 1969; Meycr \& Meyer, 1977). Their findings are consistent in that even simple blackwhite discrimination learning becomes a very difficult task when vision is restricted to the uncrossed retinal input.

Since enuclcation of one eye at birth results in the increase in the number of functionally effective optic terminals in the LGB, these findings made us wonder if such a rat might show a better performance in black-white discrimination learning. There are at lcast two methods to test for this possibility: One is to compare the learning rates of the discrimination task after performing unilatcral visual cortex lesion contralateral to the remaining eve between rats with one eye removed at birth and those cnucleated at maturity, and the other is to compare the retention rates of the habit learned with both the visual cortices intact after unilateral visual cortex lesion between the two kinds of rats. The present experiment cmployed the latter method.

\section{Methods}

\section{Subjects and Apparatus}

Thirty-eight male albino rats of the Wistar Strain, weighing $350-550 \mathrm{~g}$ when training began, were used as subjects. The right eye of 20 rats was enucleated at birth (within $24 \mathrm{hrs}$ after the time of birth) after which they were reared in an usual light-dark environment until three months of age (OEB group), while the other rats had their right eyes removed at three months of age. Of the latter group, eight rats were trained for discrimination task one week after the eye enucleation (Control group 1 ), and ten were reared for additional three months (Control group 2). Control group 2 was made to test for if the period of three months' rearing with one eye after three months of age has a similar effect on the discrimination learning as does the same condition set immediately after birth. Enucleation of the eye was performed under ether anesthesia.
A training apparatus was a modified shockavoidance discrimination box originally devised by Thompson and Bryant (1955). It consisted of start, choice and goal compartments and had two stimulus doors $(12 \times 12 \mathrm{~cm})$ which were set $10 \mathrm{~cm}$ apart into the wall between the choice and goal compartments and which were separated by a partition (18 cm long). Three fluorescent lamps of $10 \mathrm{~W}$ each positioned above the choice compartment were the only source of illumination of the room in which the training was conducted.

\section{Training}

The rats were handled $10 \mathrm{~min}$ daily for two days after which the training took place. The method was as follows. On the first day, the rats were permitted to explore the apparatus for $30 \mathrm{~min}$ with all doors taken out. On the second day, the animals were placed within the start box, were briefly shocked $(50 \mathrm{~V}, \mathrm{AC})$ unless they moved into the choice compartment within $5 \mathrm{sec}$, and received a second shock within the choice compartment if they failed to exit through a goal box opening within $30 \mathrm{sec}$. This procedure was repeated until the animals made five successive runs into the goal box without receiving shocks at any place. A pair of gray cards were then hung down with a slight angle away from the rat between the choice compartment and the goal box, and the rats were run until they made three consecutive avoidances of shock by exiting within $30 \mathrm{sec}$. Following this, the same gray doors were hung straight down and the animals were again run until they made five consecutive avoidances. The black-white discrimination training took place on the third day. This training procedure was basically the same as that mentioned above except that the rats got shocked when they gained access to the nagative (black) door. The animals were trained 20 trials a day until they reached the criterion of two successive blocks of ten trials in each of which nine avoidances were made. The position of the positive (white) door was varied according to a Gellerman series (Gellerman, 1933). After every trial the stimulus doors were washed in order to minimize the pos- 
sibility that the rats might use non-visual (i.c. olfactory) cues.

\section{Corlical Lesion and Retraining}

Following attainment of the criterion on the original discrimination learning, 15,6 and 8 subjects of the OEB, Control 1 and Control ? groups respectively received lesion in the visual cortex contralateral to the remaining eye (CT lesion). The remaining 5,2 and 2 rats of the corresponding three groups received lesion in the visual cortex ipsilateral to the remaining eye (IP lesion). The cortical lesion was accomplished by aspiration under sodium pentobarbital anesthesia $(50 \mathrm{mg} / \mathrm{kg}$, ip.). The lesion was intended to extend from the tip of the occipital pole to $7 \mathrm{~mm}$ anteriorly and from midline to $7 \mathrm{~mm}$ laterally. This lesion included all of the areas 17,18 and $18 \mathrm{a}$ (Krieg, 1946), and all of the primary and extraprimary visual cortex delineated electrophysiologically (Adams \& Forrester, 1968; Montero, 1973); hence the areas receiving projection from the LGB based on the histological data (Hughes, 1977; Ribak \& Peters, 1975).

After a recovery of ten days, the rats were retrained on the same task to the same criterion previously mentioned. Retention rate as measured by a savings score was then determined on each rat using the formula, Savings Score $=100 \times(X-Y) / X$, where $X$ represents trials to criterion in original learning and $Y$ trials to criterion in relearning.

\section{Test for Cortical Dependency of Relearning}

Altogether ten rats of the OEB group and eight of the Control group 2 with the CT lesion were employed for this test. Seven subjects of the OEB group and six of the Control group 2 underwent surgery by which the still functional visual cortex ipsilateral to the remaining eye was destroyed. The rest of both groups had sham operation. The lesion technic was the same as that described previously. The rats were again allowed to recover from surgery for ten days, and they were tested for retention of the task as measured by re-relearning.

\section{Histology}

After relearning or re-rcleaming was completed, each rat was sacrificed under ether. The brain was taken out and fixed with 10\% formalin for at least one week. The dorsal surface of the brain was photographed and the extent of the cortical lesion was drawn based on a Krieg's brain surface map (Krieg, 1946). The brain was then frozen and sectioned frontally at $30 \mu \mathrm{m}$ through the LGB, and every 6 th section was stained with cresyl violet. Subsequently, the amount of retrograde degeneration in the dorsal LGB was assessed and drawn based on a König and Klippel's atlas (König \& Klippel, 1963).

\section{Results}

\section{Anatomical Findings}

Figures 1 through 4 show the extent of the CT and IP lesions as well as the lesion made after relearning for each subject. Also included in the figures are estimates of the amount of retrograde degeneration of the respective $\mathrm{LGB}$ in three coronal levels. From the top, they present the rostral, middle and caudal sections respectively. The degenerated regions and the extent of the cortical lesions are depicted in black.

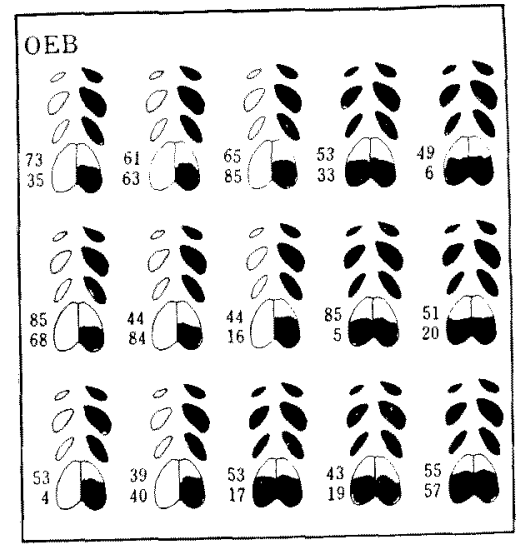

Fia. 1. The extent of the CT lesion made after original leaming and of the additional IP lesion made after relearning, and representation of degeneration in the respective LGB for each subject in the OEB group. 
As seen in these figures, the shrinkage of the LGB was observed on the side contralateral to the enucleation only at birth. There was localized sparing in 5,1 and 2 rats of the OEB, Control 1 and Control 2 groups with the CT lesion, and in 2 and 1 rats of the OEB and Control 1 groups with the IP lesion. In both lesions, MannWhitney $U$ test revealed no significant difference of savings scores between subjects with complete degeneration and those with localized sparing (CT lesion: $U=84$; IP lesion: $U=8$ ). Consequently, all subjects with localized sparing were included in the corresponding groups for further analyses.

\section{Behavioral Results}

The trials required by the individual

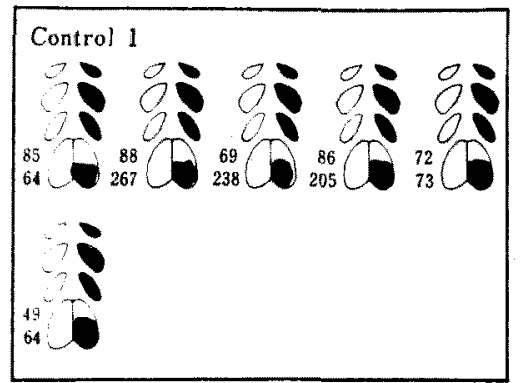

Frg. 2. The extent of the GT lesion made after original learning, and representation of degeneration in the respective LGB for each subject in the Control group 1.

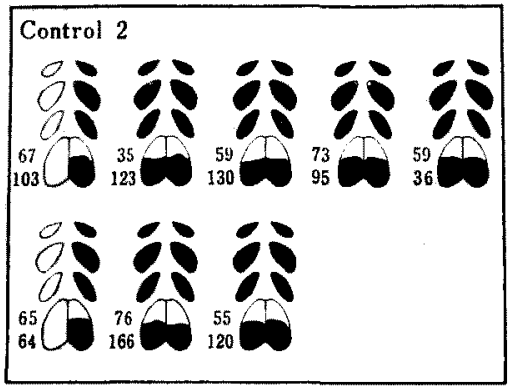

FIG. 3. The extent of the CT lesion made after original learning and of the additional IP lesion made after relearning, and representation of degeneration in the respective LGB for each subject in the Control group 2. subject to learn and to relearn the blackwhite discrimination problem are given with the brain diagrams in Figs. 1 through 4. The learning scores are given at the top, and the relearning scores at the bottom.

Preoperatively, the OEB group learned the discrimination task to criterion in $\mathbf{5 4 . 0}$ (median) trials, while the Control 1 and Control 2 groups learned it in 78.5 and 62.5 trials respectively. A Kruscal-Wallis analysis of trials to reach the criterion indicated a significant difference among the three groups $(H=6.92, d f=2, p<.05)$. Further analyses by $U$ test revealed that the Control group 1 learned significantly more slowly than the OEB group ( $U=$ $127.5, p<.05)$.

After reaching the criterion, all subjects of the three groups received a lesion in the visual cortex either ipsilateral or contralateral to the side of the remaining eye. These animals were retrained on the same task to a criterion of two consecutive 9/10 correct responses. When the IP lesion was made, the OEB, Control 1 and Control 2 groups showed the median savings scores of $78.2,86.4$ and $92.8 \%$ respectively. A Kruscal-Wallis analysis showed

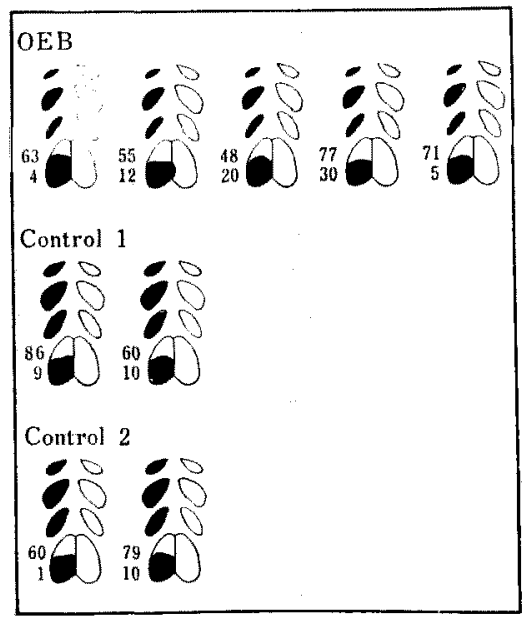

Fig. 4. The extent of the IP lesion made after original learning and representation of degeneration in the respective LGB for each subject in the $\mathrm{OEB}$, Control 1 and Control 2 groups. 
that there was no significant difference in the savings scores $(H=1.49)$, which indicated that the three groups were not different from one another with respect to the amount of retention. When pooled, the data of the three groups yielded a median savings score of $87.3 \%$ as shown in Fig. 5.

The CT lesion, however, had differential effects on the rate of relearning in the three groups as seen in Fig. 5. The median savings scores of the OEB, Control 1 and Control 2 groups were 52.1, -84.5 and $-86.0 \%$ respectively. The savings scores were found to be significantly different among the three groups (KruscalWallis test, $H=11.31, d f=2, \quad p<.005$ ). Mann-Whitney $U$ tests established that the OEB group differed from either control group (Control 1: $U=13, p<.05$; Control 2: $U=15, p<.01)$, whereas these two control groups did not differ from each other. That is, the OEB group retained the habit in the amount which is yet smaller than that of all the subjects receiving the IP lesion $(U=111, p<.05)$, but the Control 1 and Control 2 groups retained nothing and took approximately twice as much training as required in the original learning. These latter findings suggest that the period of three months with one eye provided after three months of age

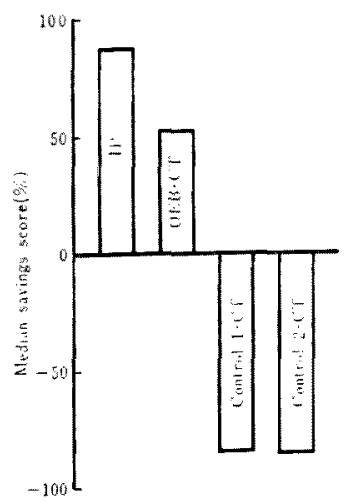

FIG. 5. The median savings score of all the subjects receiving the IP lesion and those of subjects receiving the CT lesion in the OEB, Control 1 and Control 2 groups.
TABLe 1

Median correct responses in last 20 trials of relearning (RL) and in first 20 trials of rerelearning (RRL) in subjects of both OLB and Control 2 groups with additional IP lesion or sham operation

\begin{tabular}{lcccc}
\hline \multirow{2}{*}{ Groups } & \multicolumn{2}{c}{ Additional lesion } & \multicolumn{2}{c}{ Sham operation } \\
\cline { 2 - 5 } & RL & RRL & RL & RRL \\
\hline OEB & 18.0 & $11.0^{*}$ & 18.0 & 18.0 \\
Control 2 & 18.0 & $11.0^{* *}$ & 18.0 & 17.0 \\
$* p<.01$ & $* * p<.025$ & & \\
\end{tabular}

does not have any positive effect on relearning of the habit as seen in the $\mathrm{OEB}$ group.

Table 1 summarizes the results of the test for cortical dependency of relearning. Cortical dependency was specifically tested by comparing the number of correct responses made in the last 20 trials of relearning and those made in the first 20 trials of re-relearning. It was found that by the additional IP lesion the occurrences of correct response became significantly less frequent in both the $\mathrm{OEB}$ and Control 2 groups (sign test), and that although there were only 3 rats in the former group and 2 rats in the latter group which received sham operation, all 5 rats showed complete retention. Cihi-square tests on the number of correct responses in the rerelearning condition revealed that the probability of correct performance dropped to a chance level after the additional IP lesion in each of all 13 subjects of both the OEB and Control 2 groups $\left(\chi^{2}=.10-\right.$ .92). Of the two control groups, the reason for not including the Control group 1 for this test is that the Control 1 and Control 2 groups did not show any reliable difference in the savings scores after the CT lesion and that there was no reason to suppose that different neural mechanisms should participate in bringing out the similar relearning rates in these two groups. 


\section{Discussion}

As shown in Fig. 5, when the CT lesion was made, and thus only the uncrossed relinal input could reach the visual cortex, the median savings score of the OEB group was far superior to those of Control 1 and Control 2 groups, and it was the only positive score. These results clearly show that the OEB group retained the habit to some extent but the two control groups did not. The degree of retention of the OEB group in this condition, howcver, was about half of that obtained when the crossed retinal input could reach the visual cortex. But the fact that a three month experience with one eye after three months of age (Control group 2) did not cnhance the retention suggests that the phenomenon is rather specific to eye enucleation at birth.

As already mentioned, one eye enucleation at birth results in the increase in the number of uncrossed optic nerve terminals in the LGB and SC (Lund et al., 1973) and the same treatment greatly potentiates a postsynaptic component in the LGB upon stimulation of the ipsilateral optic nerve (Sakai, 1978). These findings lead us to interpret the partial retention of the habit in the OEB group receiving the CT lesion as a result of the increase in the uncrossed retinal input to the structures which are essential to the performance of the black-white discrimination habit.

Thompson, Truax and Thorne (1970) has suggested that the route from the retina to the visual cortex and then down to the subcortical structures such as the posterior thalamic nucleus and red nucleus is essential for the performance of the discrimination habit. The present results are generally in agreement with their view that the visual cortex is somehow involved in this type of learning. It should be noted however that destruction of the visual cortex receiving a massive crossed retinal input (CT lesion) had a far greater effect on the loss of the habit than that of the visual cortex receiving a small amount of uncrossed retinal input (IP lesion) as shown in the difference in their respective savings scores. It should also be emphasized that the relearned habit with the CT lesion was again lost after the additional IP lesion. This indicates that even in a situation where only a small amount of uncrossed retinal input reaches the visual cortex, the route to the visual cortex and then to its related subcortical structures plays an important role in the performance of the visual habit.

It is thus reasonable to assume that in the original learning of the task with both visual cortices intact the uncrossed retinal input must participate in establishing engrams through the visual cortex. The positive savings score of the OEB group after the CT lesion suggests that the uncrossed retinal input was indeed effective in forming engrams, but the negative savings scores of both control groups suggest it was not. The reason for ineffectiveness in the latter two groups is probably that engrams can not be formed through the small number of uncrossed optic fibers in the amount of time required for engrams to be formed through the massive crossed retinal input. Of course, this does not mean that the small amount of uncrossed retinal input in the two control groups are functionless, because relearning of the task after the CT lesion was still possible if two to three times as much training was provided (see Figs. 2, $3 \&$ 5). Therefore, the difference in the savings scores after the CT lesion between the OEB and the two control groups is considered mainly due to the difference in the amount of uncrossed retinal input feeding into the visual cortex and then into the related subcortical structures during the original learning.

A question then arises as to the route from the retina to the visual cortex. There are at least two possible routes. One is from the retina to the LGB and then to the visual cortex. The other is from ret- 
ina to the SC to the lateral posterior thalamus and to the visual cortex. However, a number of studies have already demonstrated that while the visual cortex lesion leading to a total degeneration of the LGB results in an almost complete loss of a black-white discrimination habit, the SC. lesion does not (c... Thompson, 1960). It is therefore likely that a certain amount of retention of the habit seen in the OEB group after the CT lesion is mostly dependent upon engrams made by the input from the LGB.

Then, it may be expected that there be differences in the original learning rates among the three groups. However, the median trials required to reach criterion are $54.0(\mathrm{OEB}), 78.5$ (Control 1) and 62.5 (Control 2) respectively; there was no reliable difference in the learning rates between the OEB group and the Control group 2 which received the same experimental treatment as the OEB group except for the time of enucleation. One explanation is that in the original learning of the habit a massive crossed retinal input can be fed into the visual cortex contralateral to the remaining eye and this contribution is so dominant in making the habit that that of the retinal input ipsilateral to the remaining eye is masked. A better way to test this speculation is to compare the original learning rates of the OEB, Control 1 and Control 2 groups after making the lesion in the visual cortex contralateral to the remaining eye.

In the trials required to reach criterion in the original learning, why did the Control group 1 learn more slowly than the OEB and Control 2 groups? It was speculated that the difference might come from the amount of time provided for the former and the latter two groups after enucleation of one eye before the discrimination training took place. In other words, it would take some time for the rat to be able to move around freely using only one eye: The animals of the OEB and Control 2 groups had enough time, whercas those of the Control group I did not. If so, such effects should be reflectod in the tendency in choosing the right and left doors in the choice comparment in the initial stage of training. This was tested by comparing the numbers of left door choices made in the first 20 trials among the three groups. The median numbers of left door choices were $8.5,4.5$ and 4.5 for the OEB, Control 1 and Control 2 groups respectively. Although the subjects of the three groups tended to choose the right door more often than the left door, their choice tendencies were not different from one another (KruscalWallis test, $H=.31$ ). Therefore the difference in time after enucleation of one eye can not account for the obtained results. At present we do not know the reason.

In summary, the rats with one eye removed at birth can form some engrams for the black-white discrimination habit somewhere in the brain through the visual cortex ipsilateral to the remaining eye, whereas those with one eye enucleated at three months of age can not. These results can be considered as behavioral counterparts to the histological and electrophysiological changes in the visual pathway produced by enucleation of one eye at different ages.

\section{REFERENCES}

AnAms, A. D., \& Forrester, J.M. 1968 The projection of the rat's visual field on the cerebral cortex. Quarterly Journal of Experimental Physiology, 53, 327-336.

Creer, D. J., \& Sheridan, G. L. 1966 Monocular acquisition and interocular transfer in albino rats with unilateral striate ablations. Psychonomic Science, 6, 89-90.

Gelilerman, L. W. 1933 Chance orders of alternating stimuli in visual discrimination experiments. Journal of Genetic Psychology, 42, $207-208$.

Hrckey, T. L. 1975 Translaminar growth of axons in the kitten dorsal lateral geniculate nucleus following removal of one eye. Journal 
of Comparative Neurology, 161, 359-382.

Hughes, H. C. 1977 Anatomical and neurobehavional investigations concerning the thalamocortical organization of the rat's visual system. Journal of Comparative Neurology, 175, 311-336.

KöNtG, J.F.R., \& KI.lPPEL, R. A. 1963 The rat brain: A stereolaxic atlas of the forebrain and lower parts of the brain stem. Baltimore: Williams \& Wilkins.

Krter, W.J.S. 1946 Accurate placement of minute lesions in the brain of the albino rat. Quarterly Bulletin, Northwestern University Mtedical School, 20, 199-208.

Lund, R. D. 1965 Uncrossed visual pathways of hooded and albino rats. Science, 149, 15061507.

Lund, R. D., Cunnmoham, T.J., \& Lund, J. S. 1973 Modified optic projections after unilateral eye removal in young rats. Brain, $B e$ havior and Evolution, 8, 51-72.

MIrite, D. R., \& Mever, P. M. 1977 Dynamics and bases of recoveries of functions after injuries to the cerebral cortex. Physiological Psycholog $y, 5,133-165$.

Montero, V. M. 1973 Evoked responses in the rat's risual cortex to contralateral, ipsilateral, and restricted photic stimulation. Brain Re- search, 53, 192-196.

Nadel, L., \& Buresová, O. 1969 Interocular transfer in the hooded rat. Physiology and $B e-$ havior, 4, 613-619.

Ribak, C. E., \& Peters, A. 1975 An autoradiographic study of the projections from the lateral geniculate body of the rat. Brain Research, 92, 341-368.

SAKAl, M. 1978 Reorganization of the visual system following removal of one eye in the rat: On the electrical activity of the lateral geniculate body. Proceedings of The 42 nd Annual Convention of the Japanese Psychological Association, 140-141. (in Japanese)

Thompson, R. 1960 Retention of the brightness discrimination following neo-cortical damage in the rat. Journal of Comparative and $P h y^{\prime}$ siological Psychology, 53, 212-215.

Trompson, R., \& Bryant, J. H. 1955 Memory as affected by activity of the relevant receptor. Psychological Report, 1, 393-400.

Thompson, R., Truax, T., \& Thorne, M. 1970 Retention of visual learning in the white rat following knife-cuts through the posterior thatamus and ventromedial midbrain. Brain, Behavior and Evolution, 3, 261-284.

(Reccived Oct. 5, 1979) 